\title{
Effects of a nine-strain bacterial synbiotic compared to simethicone in colicky babies - an open-label randomised study
}

\author{
J. Piątek $^{1^{*}}$, M. Bernatek ${ }^{1}$, H. Krauss ${ }^{1}$, M. Wojciechowska ${ }^{2}$, Z. Chęcińska-Maciejewska ${ }^{3}$, P. Kaczmarek ${ }^{1}$ and \\ H. Sommermeyer ${ }^{1}$ \\ ${ }^{1}$ Department of Medicine, The President Stanisław Wojciechowski State University of Applied Sciences in Kalisz, Nowy \\ Šwiat 4, 62-800 Kalisz, Poland; ${ }^{2}$ Mother and Child Health Department, Karol Marcinkowski Medical University in Poznań, \\ ul. Fredry 10, 61-701 Poznan, Poland; ${ }^{3}$ Physiology Department, Karol Marcinkowski Medical University in Poznań, ul. \\ Fredry 10,61-701 Poznan, Poland; drpiatek@interia.eu
}

Received: 10 August 2020 / Accepted: 3 December 2020

(c) 2021 Wageningen Academic Publishers

OPEN ACCESS (1) RE(D) REARCH ARTICLE

\begin{abstract}
The aim of the study was to determine effects of administration of simethicone and a multi-strain synbiotic on the crying behaviour of colicky babies. The study design consisted of an open-label, two parallel treatment group study involving 87 infants aged 3-6 weeks with infantile colic (defined as crying episodes lasting 3 or more hours per day and occurring at least 3 days per week within 3 weeks prior to enrolment) randomly, unequally [1:1.5] assigned to receive simethicone $(n=33)$ or a multi-strain synbiotic $(n=54)$ orally for 4 weeks. The multi-strain synbiotic contained Lactobacillus acidophilus LA-14, Lacticaseibacillus casei R0215, Lacticaseibacillus paracasei Lp-115, Lacticaseibacillus rhamnosus GG, Ligilactobacillus salivarius Ls-33, Bifidobacterium lactis Bl-04, Bifidobacterium bifidum R0071, Bifidobacterium longum R0175 and fructooligosaccharides). Primary outcome measures were the responder rates (effect $\geq 50 \%$ reduction from baseline) of the measures 'crying days last 3 weeks', 'average evening crying duration last 3 weeks' and 'reduction of average number of crying phases per day last three weeks' at the end of treatment. The study is registered at ClinicalTrials.gov under NCT 04487834. Significantly higher responder rates (effect $\geq 50 \%$ reduction from baseline) of the multi-strain synbiotic compared to simethicone were found for the measures 'crying days last 3 weeks' $(72 \%$ vs $18 \%, P<0.0001)$ and 'average evening crying duration last 3 weeks' ( $85 \%$ vs $39 \%, P=0.0001$ ). No significant difference was found for the measure 'reduction of average number of crying phases per day last three weeks' ( $50 \%$ vs $42 \%, P=0.4852)$. No adverse effects were reported for the two treatment groups. Based on these results, the multi-strain synbiotic can be considered as an interesting therapeutic possibility for the treatment of infantile colic, worthwhile to be investigated further in non-clinical and clinical studies.
\end{abstract}

Keywords: infantile colic, extensive crying, gut microbiota, probiotic

\section{Introduction}

Infantile colic or extensive crying is a major burden for newborns, their parents and healthcare providers (Landgren and Hallström, 2011). In most of the cases infantile colic will disappear after the first three to five months of life (Sung, 2018). Nevertheless, due to its stressful nature for parents, infant colic is among the leading causes to consult a health care professional during early infancy (Lucassen, 2010). The parental burden associated with infantile colic finally results in enormous pressure on paediatricians to prescribe at least some kind of remedy. Diagnosis of infantile colic can be based on the Wessel's criteria, defined by crying and restlessness for more than $3 \mathrm{~h}$ a day for more than three days per week for more than three weeks (Wessel et al., 1954). Depending on diagnostic details, occurrence rates between 3 to $40 \%$ of all infants have been found (Helseth and Begnum, 2002; Lucassen et al., 2001).

The aetiology of infant colic remains unclear (Sarasu et al., 2018), with a variety of potential causes (gastrointestinal, hormonal, neurodevelopmental, and psychosocial) 
discussed. This has resulted in a broad range of therapeutic approaches aiming to address the problem (Lucassen, 2010; Mai et al., 2018).

Simethicone (Anonymous, nd), a mixture of dimethicone and $\mathrm{SiO}_{2}$, is a rather old product that, it is claimed, acts as a topical barrier for protecting the gut mucosa against irritants. It is not absorbed and is virtually non-toxic. While its use in diagnostic procedures is well-established, the therapeutic effects in a number of gastroenterological indications are contradictory (Meier and Steuerwald, 2007). In infantile colic, a number of smaller simethicone trials (Danielsson and Hwang, 1985; Metcalf et al., 1994; Sethi and Sethi, 1988) have been published, but evidence did not reach the threshold of significance (Biagioli et al., 2016). Despite the absence of evidence for beneficial effects, simethicone is widely used for the treatment of infantile colic in some countries.

There is growing evidence that the gut microbiotas of colicky infants significantly differ from those of noncolicky babies (Savino et al., 2004, 2005, 2009). In the gut microbiota of colicky babies a lower level of commensal bacteria like Lactobacilli and Bifidobacteria and higher numbers of Proteobacteria have been found. Among these Proteobacteria were Escherichia and Klebsiella bacteria (Savino et al., 2011), which are well known for their gasproducing properties, as well as the potential production of inflammatory lipopolysaccharides (LPS). In addition, it has been described that the gut microbiota of colicky infants exhibits a slower bacterial colonisation, a reduced microbiota diversity and a lower microbiota stability (De Weerth et al., 2013). These findings have triggered a number of studies investigating the effect of supplementation of the gut microbiota of colicky babies with products containing probiotic bacteria without a prebiotic component (probiotics), or with (synbiotics).

Although infantile colic is in most cases a self-limiting condition, paediatricians are writing a prescription for most of the affected infants. Unpublished results from surveys, performed by our research team among German and Polish paediatricians, revealed that in these two countries simethicone and pro-/synbiotics were the most frequently prescribed products for infantile colic. The objective of the present study was to compare the effects of simethicone with those of a food supplement multi-strain synbiotic on the crying behaviour in colicky babies. This multi-strain synbiotic preparation, which is frequently used by German and Polish paediatricians, contains one Lactobacillus (Lactobacillus acidophilus), three lacticaseibacilli (Lacticaseibacillus casei, Lacticaseibacillus paracasei, Lacticaseibacillus rhamnosus GG), one Lactiplantibacillus (Lactiplantibacillus plantarum), one Ligilactobacillus (Ligilactobacillus salivarius), three bifidobacteria (Bifidobacterium lactis, Bifidobacterium bifidum, Bifidobacterium longum) and the prebiotic fructooligosaccharides (FOS). In a study, recently published by us (Piatek et al., 2020), it was found that this multi-strain synbiotic antagonises the in vitro growth of the pathogenic bacteria Escherichia coli EPEC, Shigella sonnei, Salmonella typhimurium, Klebsiella pneumoniae and Clostridioides difficile.

During the preparation phase of this study, we learned that pediatricians willing to participate were not ready to treat colicky babies with a placebo, as this would not be acceptable for the majority of parents of the colicky babies. In addition, placebo preparations were neither available for us for simethicone nor for the multi-strain synbiotic. Consequently, our study had to be performed as an open trial, comparing the two products without a placebo control group. Effects of simethicone in infantile colic have been shown not to differ significantly from treatment with a placebo (Danielsson and Hwang, 1985; Metcalf et al., 1994; Sethi and Sethi, 1988), therefore the simethicone treatment group in our study has to be considered as the control group against which the effect of the multi-strain synbiotic has to be evaluated.

\section{Materials and methods}

This was an open-label, unequally randomised [1:1.5], two treatment group study conducted in Poland during April and July 2020. The trial was registered at ClinicalTrials.gov (NCT 04487834). Parents of all infants gave their informed consent for participation before the infants were included in the study. The study was conducted in accordance with the Declaration of Helsinki, and the protocol was approved by the Ethics Committee of The President Stanisław Wojciechowski State University of Applied Sciences in Kalisz (Project identification code 1/2020). Assuming a significance level of $5 \%$, a power of $80 \%$, a responder rate of $50 \%$ in the simethicone group and of $80 \%$ in the multi-strain synbiotic group and a dropout rate of $0 \%$, a required sample size of 36 patients was calculated by using the Sample Size Calculator of Sealed Envelope Ltd. (London, UK). Recruitment for the study was carried out in hospitals in Poznan, Poland. Infants (aged 3-6 weeks) of parents who agreed were assessed for eligibility to participate in the study. After organic causes of crying were carefully excluded, parents, supported by paediatricians, midwives or nutritionists, filled out a questionnaire to obtain data concerning type of delivery, birth weight, gestational age, type of feeding and details of the crying behaviour of the babies. Babies who fulfilled the Wessel criteria (Wessel et al., 1954) of extensive evening crying for at least $3 \mathrm{~h}$ per day, during at least three days per week, during the last three weeks, were included in the study. Exclusion criteria were prior treatment with probiotics, synbiotics or antibiotics. The study population comprised breastfed and formulafed, as well as mixed-fed new-borns. 
Babies diagnosed to have infantile colic were randomly assigned to two treatment-groups. A two-treatment unequal allocation [1:1.5] randomisation scheme was generated by using the Simple Randomiser of Sealed Envelope Ltd. Product was allocated to patient by paediatrician using the randomisation scheme based on entry into the study. One group was treated for four weeks with simethicone (Espumisan ${ }^{\ominus} 100 \mathrm{mg} / \mathrm{ml}$, Berlin-Chemie/Menarini Polska Sp. z o.o., Warsaw, Poland). Simethicone was administered 3-6 times per day with each treatment comprising 6 drops of the $100 \mathrm{mg} / \mathrm{ml}$ emulsion. The second group was treated with one stick pack of a multi-strain synbiotic (Multilac ${ }^{\circ}$ Baby, Vivatrex GmbH, Aachen, Germany) per day. Each stick pack of Multilac Baby contains a total of $10^{9} \mathrm{cfu}$, with equal cfu amounts of the following probiotic bacteria: $L$. acidophilus LA-14, L. casei R0215; L. paracasei Lpc-3; L. plantarum Lp-115; L. rhamnosus GG, L. salivarius Ls-33, B. lactis Bl-04, B. bifidum R0071, B. longum R0175 and 1.43 $\mathrm{g}$ of the prebiotic FOS. Parents were provided with a diary (24 h Parental Daily Report) to protocol crying behaviour, drug administration and side effects.

After the end of the treatment period changes in crying behaviour were assessed with the same set of questions used for the enrolment into the study. In addition, it was investigated if antibiotics were administered during the treatment period. Numerical measures (days of crying during the last three weeks, duration of evening crying, average number of crying phases per day) were analysed by calculation of the average and the standard deviation. In addition, the rate of treatment success was determined, which was defined as a reduction of a given measure (days of crying during the last three weeks, average duration of evening crying during last three weeks, average number of crying phases per day during last three weeks) by equal or more than $50 \%$, compared to the value at the time of enrolment (Sung, 2018). For non-numerical measures (questions answered either by yes or no), the percentages of 'yes' (or 'no') answers were calculated.

The statistical analyses were conducted with GraphPad Prism software version 8.2 (GraphPad Software, San Diego, CA, USA) or MedCalc Statistical Software version 19.2.1 (MedCalc Software Ltd., Ostend, Belgium). The Student t-test was used to compare mean values of continuous variables approximating a normal distribution. For nonnormally distributed variables, the Mann-Whitney U test was used. The X2 test or Fisher exact test was used, as appropriate, to compare percentages. The MedCalc Statistical Software version 19.2.1 was used to calculate the relative risk (RR) and number needed to treat (NNT), all with a 95\% confidence interval (CI). The difference between the treatment groups was considered significant when the $P$-value was less than 0.05 , when the $95 \% \mathrm{CI}$ for RR did not include 1.0, or when the $95 \% \mathrm{CI}$ for mean difference did not include 1.0, or when the $95 \%$ CI for mean difference did not include 0 . One-way ANOVA followed by Dunnett's multiple comparisons test as well as Cochran-Armitage Chi-squared test for trend were performed using GraphPad Prism software. All statistical tests were two-tailed and performed at the $5 \%$ level of significance. All analyses were conducted on an intention-to-treat basis, including all patients in the groups to which they were allocated.

\section{Results}

Figure 1 shows the flow of patients through the study. During the recruitment period of the study a total of 915 babies were born. Parents of 211 babies agreed to have their infants assessed for eligibility to participate in the study. Of these, 87 (41.2\%) fulfilled the Wessel's criteria used for enrolment. One baby was fulfilling the Wessel's criteria of enrolment but was excluded because of a previous antibiotic treatment. 123 new-borns were excluded for not fulfilling the Wessel's criteria. Of the babies diagnosed for infantile colic, 33 were treated with simethicone and 54 with the multi-strain synbiotic. No patient was lost or had to be excluded during the remaining phase of the trial. Table 1 shows the baseline characteristics of both treatment groups. Statistical analyses revealed that there were no significant differences between the two treatment groups.

Results for the three main measures of the study: (1) number of days of crying during the last three weeks, (2) average duration of evening crying during the last three weeks and (3) average number of crying phases per day during the last three weeks are shown in Figure 2. As is visible in Figure $2 \mathrm{~A}$ and $2 \mathrm{~B}$, simethicone and the multi-strain synbiotic treatment caused a significant reduction in both the number of days of crying during the last three weeks and the average duration of evening crying during the last three weeks. Comparing the effects of the multi-strain synbiotic with that of simethicone revealed that the multi-strain synbiotic had significantly $(P \leq 0.0001)$ superior effects on these two main measures. The average number of crying phases during the last three weeks was significantly reduced by the multistrain synbiotic, but not by simethicone (Figure 2C).

Treatment success rates for the reduction of days of crying during the last three weeks and for the reduction of average evening crying duration last three weeks were significantly higher in the multi-strain synbiotic group than in the simethicone group (Table 2). The treatment success rate for the reduction of average number of crying phases during the last three weeks showed no significant difference between simethicone and the multi-strain synbiotic treatment groups.

In addition to the main measures, a number of other crying behaviour observations were assessed by the study (Table 3). In order to verify if the effects of the multi-strain synbiotic were significantly different from those of simethicone, a 


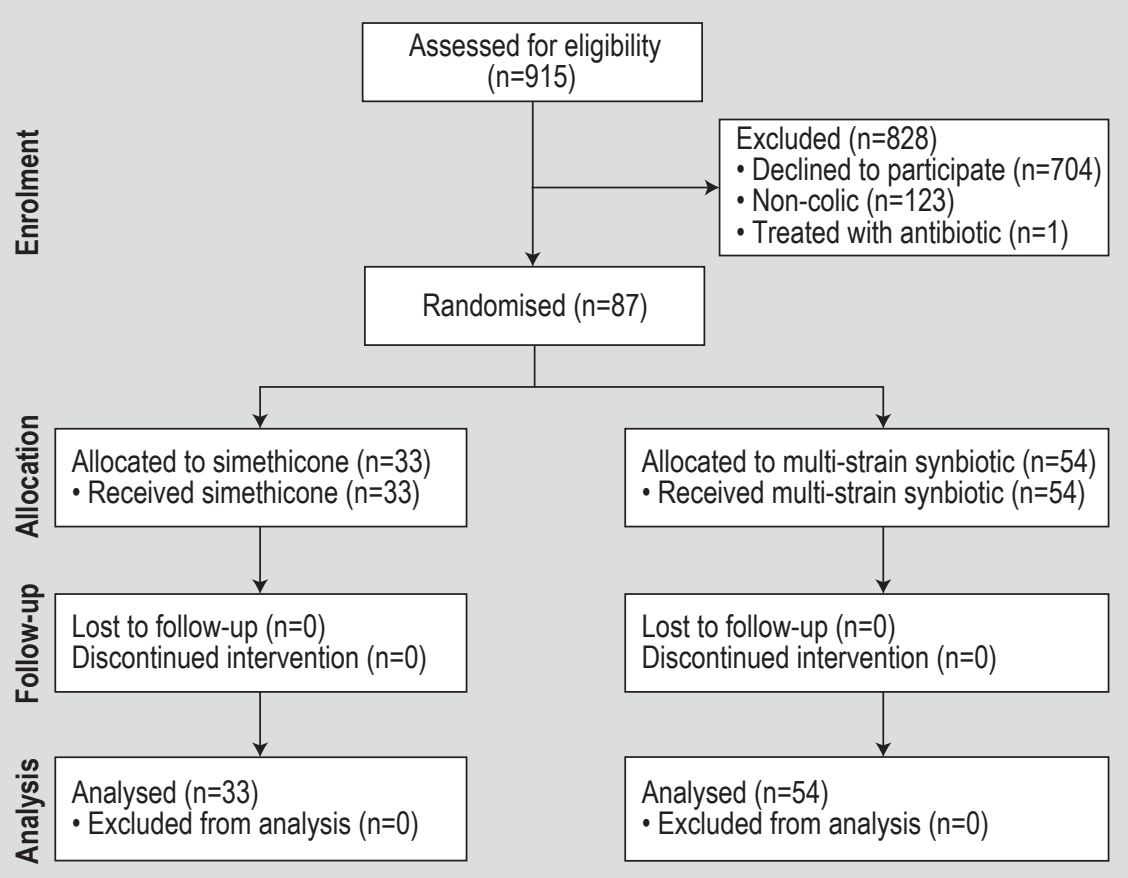

Figure 1. Patient enrolment and study progress.

Table 1. Baseline characteristics of simethicone and multi-strain synbiotic treatment groups. ${ }^{1}$

\begin{tabular}{llll} 
& Simethicone $(\mathbf{n = 3 3 )}$ & Multi-strain synbiotic $(\mathbf{n}=\mathbf{5 4})$ & $P$-value ${ }^{2}$ \\
Gender (F/M) & $18 / 15$ & $29 / 25$ & $0.9394^{\mathrm{a}}$ \\
Type of delivery (normal/caesarean) & $24 / 9$ & $39 / 15$ & $0.9594^{\mathrm{a}}$ \\
Birthweight (g) & $3,480 \pm 412$ & $3,500 \pm 404$ & $0.8246^{b}$ \\
Feeding (breast/formula/mixed) & $22 / 11 / 0$ & $27 / 17 / 10$ & $0.0943^{\mathrm{a}}$ \\
Days of crying last 3 weeks (days) & $18.2 \pm 1.7$ & $18.3 \pm 2.1$ & $0.8246^{b}$ \\
Duration of evening crying (h/day) & $2.7 \pm 0.5$ & $2.8 \pm 0.7$ & $0.4760^{\mathrm{b}}$ \\
\hline${ }^{1}$ Data are expressed as average \pm standard deviation or numbers. & & \\
${ }^{2}$ Statistical tests indicated by superscript letter (a) Chi-squared test, and (b) Student t-test. & &
\end{tabular}

Cochran-Armitage Chi-squared test for trend was used. The linear trend of data ordered by treatment efficiency was tested. Data were ordered in three groups: before treatment $(\mathrm{n}=87)<$ simethicone $(\mathrm{n}=33)<$ multi-strain synbiotic $(n=54)$. In conclusion, statistically significant, greater effectiveness of the multi-strain synbiotic was confirmed for: (1) reducing the number of babies who cannot be calmed during crying, (2) reducing the number of babies with problems falling asleep, (3) reducing the number of babies with bloated belly, and (4) reducing the number of babies with retracted legs. Neither simethicone, nor the multi-strain probiotic, had an effect on the number of babies with a red face. No adverse effects were reported for the simethicone or the multi-strain synbiotic treatment group.

\section{Discussion and conclusions}

Infantile colic is a self-limiting condition and most cases are improving over time without any treatment (Lucassen, 2010). Nevertheless, for most colicky patients paediatricians in Germany and Poland are prescribing simethicone or a pro-/synbiotic (according to the unpublished results from surveys performed by our research team among German and Polish paediatricians). Despite its wide use, there is little evidence that simethicone has significant effects in infantile colic compared to a placebo treatment (Biagioli et al., 2016, Lucassen, 2010; Meier and Steuerwald, 2007). The role of probiotics in the treatment of infantile colic has been reviewed by a number of authors (Anabrees et al., 2013; Dryl et al., 2018; Ong et al., 2019; Schreck 
A

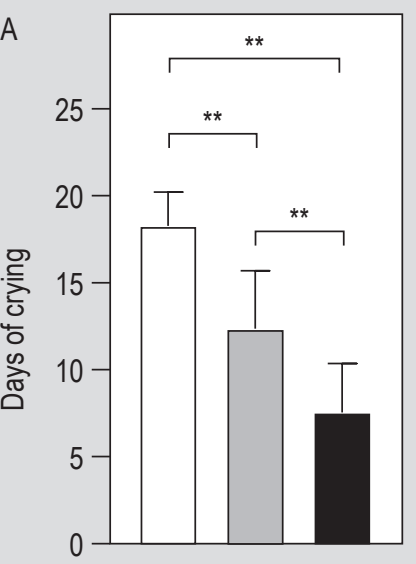

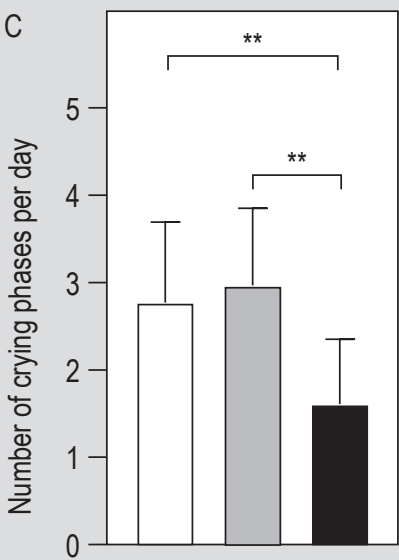

$\square$ Before treatment

Simethicone

Multi-strain synbiotic

Figure 2. Effects of simethicone or multi-strain synbiotic treatment in colicky babies on (A) days of crying during three weeks, (B) average duration of daily evening crying during three weeks and (C) average number of crying phases per day during three weeks. Results are mean \pm standard deviation. Data were analysed by one-way ANOVA followed by Dunnett's multiple comparisons test: ** $P \leq 0.0001$.

Table 2. Effect of simethicone and the multi-strain synbiotic on main measures of crying behaviour. ${ }^{1}$

\begin{tabular}{|c|c|c|c|c|c|}
\hline Outcome & $\begin{array}{l}\text { Simethicone } \\
(n=33)\end{array}$ & $\begin{array}{l}\text { Multi-strain synbiotic } \\
(n=54)\end{array}$ & $\mathrm{RR}(95 \% \mathrm{Cl})$ & NNT $(95 \%$ Cl) & $P$-value ${ }^{2}$ \\
\hline $\begin{array}{l}\text { Number of patients with treatment success: } \\
\text { reduction of days of crying } \geq 50 \% \text { ( } \% \text { responder) }\end{array}$ & $6(18 \%)$ & $39(72 \%)$ & 0.2838 & 1.427 & $<0.0001$ \\
\hline $\begin{array}{l}\text { Number of patients with treatment success: } \\
\text { reduction of avg. duration of evening crying } \geq 50 \% \\
\text { (\% responder) }\end{array}$ & $13(39 \%)$ & $46(85 \%)$ & 0.2444 & 2.184 & 0.0001 \\
\hline $\begin{array}{l}\text { Number of patients with treatment success: } \\
\text { reduction of avg. number of crying phases per day } \\
\geq 50 \% \text { ( } \% \text { responder) }\end{array}$ & $14(42 \%)$ & $27(50 \%)$ & 0.8684 & 13.200 & 0.4852 \\
\hline
\end{tabular}

Table 3. Effect of simethicone and the multi-strain synbiotic on other crying behaviour observations. ${ }^{1}$

\begin{tabular}{|c|c|c|c|c|c|}
\hline Observation & $\begin{array}{l}\text { Before treatment } \\
(n=87)\end{array}$ & $\begin{array}{l}\text { Simethicone } \\
(n=33)\end{array}$ & $\begin{array}{l}\text { Multi-strain synbiotic } \\
(n=54)\end{array}$ & $\begin{array}{l}\text { Chi-squared } \\
\text { (trend) }\end{array}$ & $P$-value ${ }^{2}$ \\
\hline Patients who cannot be calmed during crying & $50.6 \%(44)$ & $24.2 \%(8)$ & $13.0 \%(7)$ & 22.062 & $<0.0001$ \\
\hline Patients with problems falling asleep & $100.0 \%(87)$ & $57.6 \%(19)$ & $42.6 \%(23)$ & 60.499 & $<0.0001$ \\
\hline Patients with bloated belly & $62.1 \%(54)$ & $48.5 \%(16)$ & $33.3 \%(18)$ & 11.071 & 0.0009 \\
\hline Patients with retracted legs & $90.8 \%(79)$ & $69.7 \%(23)$ & $61.1 \%(33)$ & 17.150 & $<0.0001$ \\
\hline Patients with red face & $89.7 \%(78)$ & $87.9 \%(29)$ & $90.7 \%(49)$ & 0.0275 & 0.8683 \\
\hline
\end{tabular}


Bird et al., 2017; Tintore et al., 2017). Probably the most extensively investigated probiotic in infantile colic is Limosilactobacillus reuteri DSM 17938. A number of trials have been published, however, with conflicting results. Four double-blind randomised studies of breastfed infants with colic demonstrated $L$. reuteri DSM 17938 to be effective (Chau et al., 2015, Roos et al., 2013; Savino et al., 2010; Szajewska et al., 2013). In contrast, one double-blind randomised study of both breastfed and formula-fed infants with colic concluded it was ineffective even for those who were breastfed (Sung et al., 2014). A recently published individual participant data meta-analysis (IPDMA), pooling raw data from four individual studies, found that $L$. reuteri DSM 17938 had a positive effect in breastfed infants, however had no effect in formula-fed infants (Sung et al., 2018). In a single-blind randomised trial in predominantly or exclusively breastfed colicky babies, L. reuteri was found to reduce daily crying time, improve parent satisfaction and reduce maternal depression (Mi et al., 2015). In a nonblinded randomised controlled trial with breastfed babies with infantile colic, $L$. reuteri exhibited colic symptom relief superior to simethicone (Ashraf et al., 2015). L. reuteri has been demonstrated to decrease faecal calprotectin, which is a clinical marker of intestinal inflammation, and resulted in improvements of blood inflammatory markers (Savino et al., 2018).

A mono-strain probiotic containing only the probiotic strain L. rhamnosus GG is well known by paediatricians for its effects against antibiotic-associated diarrhoea (Guo and Goldenberg, 2019). When evaluated for efficacy in infantile colic, no significant treatment effects compared to placebo were found (Fatheree et al., 2016; Pärtty et al., 2015). In infants treated in tandem with behavioural support and cow's milk elimination diet, L. rhamnosus GG did not provide additional treatment effect for diary-verified colic crying, although parental report of crying suggested the probiotic intervention was effective.

Another mono-strain probiotic, Bifidobacterium animalis subsp. lactis BB-12s has been found to have positive effects in exclusively breastfed colicky infants (Nocerino et al., 2020).

There are a number of published clinical studies which investigated the effects of multi-strain synbiotics in the treatment of infantile colic. A mixture of B. lactis BB12 and Streptococcus thermophilus has been reported to reduce the incidence of infant colic (Saavedra et al., 2004). A mixture of two Bifidobacterium breve strains (BR03 and B632) was found to have no significant effects in the full population of the study. A sub-group analysis revealed that there was a significant reduction of crying time in the formula-fed group only (Giglione et al., 2016). Treatment of colicky babies with a mixture of Pediococcus pentosaceus CECT 8330 and B. longum CECT 7894 was found to increase the overall microbiota diversity and to reduce the relative abundance of Enterobacteria (mostly of Escherichia and Shigella) and Staphylococcus, compared to placebo (Tintore and Cune, 2017). In addition, a trend towards greater reduction in crying time in the probiotic group compared to placebo was observed (Santas et al., 2015). A placebo-controlled study (Kianifar et al., 2014) found a significant treatment effect (reduction of average infant crying time) in breastfed colicky babies by a synbiotic preparation containing seven probiotics (L. acidophilus, Lactobacillus bulgaricus, L. casei, L. rhamnosus, B. breve, Bifidobacterium infantis, S. thermophilus) and the prebiotic FOS. Another study (Dorreh et al., 2017) investigated the effect of a synbiotic containing three probiotic bacteria (L. reuteri, L. rhamnosus and B. infantis) and the prebiotic FOS. The study included breastfed and formula-fed colicky infants and no significant difference in crying time and the number of crying attacks between the treatment group and the control group was found.

Over recent years, we collected a number of anecdotal reports from paediatricians indicating that the multi-strain synbiotic tested in the present study had positive effects in colicky babies. Our trial is the first investigating the effects of this product in a clinical trial setting. The present study has used, as most other studies evaluating the effects of probiotics in infantile colic, the measures (1) reduction of crying days during the last three weeks, (2) reduction of the average evening crying duration during the last three weeks and (3) reduction of average number of crying phases per day during the last three weeks. Improvements in these measures are clinically and practically relevant as they are potentially translating into stress relief in the affected newborns and their parents.

The improvements observed in the simethicone treatment group of the present study would have most likely been observed as well if the colicky babies had received no treatment. However, as improvements caused by the multi-strain synbiotic were significantly larger than those of simethicone, it can be concluded that the product has treatment effects superior to a placebo treatment in infantile colic.

In addition to the superior effects on the main measures, the multi-strain synbiotic showed positive effects on some other crying behaviour observations. The number of babies who could not be calmed during crying, or who had problems falling asleep, was reduced significantly more by the multistrain synbiotic than by simethicone. These effects might be very relevant for parents of colicky babies, as not being able to calm a crying baby or getting it to sleep is experienced as utterly frustrating (Landgren and Hallström, 2011). The multi-strain synbiotic also had a significantly better effect in reducing the number of babies with a bloated belly and with retracted legs in comparison to simethicone. These 
measures of symptoms are sometimes used as indicators for infantile colic, however, treatment effects on these symptoms might not be perceived as too relevant in daily practice. In contrast, neither simethicone nor the multistrain synbiotic was found to have a significant effect on the numbers of babies with a red face.

The strength of the present study is that the effects of the multi-strain synbiotic were observed in a study with a relatively small number of participants and without selecting a subgroup of colicky babies (e.g. breast-fed babies only). Whether the treatment effects are different in certain subgroups will remain open for clarification in future larger clinical trials. A limitation of the study is the use of parental diaries to measure infants' crying behaviour. Using paper diaries (Barr et al., 1988) is not without problems (Stone et al., 2003). Nevertheless, it has been shown that paper diary recordings of common infant behaviour can provide reasonably good estimates of durations, while behavioural frequencies may be underestimated (Lam et al., 2010). Another major limitation of the study is that medication was not blinded. Past experience or non-experience with either of the two medications might therefore exhibit a bias on the results. However, this might have influenced results for some patients negatively (e.g. preference of a liquid formulation over a powder formulation), while in others it might have had a positive influence, which, at least to a certain extent, might have neutralised this bias.

While the necessity of treating this self-limiting condition can be questioned, the pressure from parents on paediatricians to prescribe is high. Results of the present study can support paediatricians in their prescribing decisions and during their communication with parents. Results of the present clinical study and of preclinical studies published earlier (Piatek et al., 2020) render the multi-strain synbiotic an interesting candidate for further clinical and non-clinical research in infantile colic.

\section{Acknowledgements}

The authors would like to acknowledge the work of Sabine Hanna from Cambridge Assessment English for proofreading, English style editing, and useful suggestions.

\section{References}

Altman, D.G., 1998. Confidence intervals for the number needed to treat. BMJ 317: 1309-1312.

Anabrees, J., Indrio, F., Paes, B. and AlFaleh, K., 2013. Probiotics for infantile colic: a systematic review. BMC Pediatrics 13: 186. https:// doi.org/10.1186/1471-2431-13-186

Anonymous, nd. Simethicone. Drugs.com. Available at: https://www. drugs.com $/ \mathrm{mtm} /$ simethicone.html.
Ashraf, M.W., Ayaz, S.B., Ashraf, M.N., Matee, S. and Shoaib, M., 2015. Probiotics are effective in alleviating infantile colic; results of a randomized controlled trial held at Benazir Bhutto Hospital, Rawalpindi, Pakistan. Rawal Medical Journal 40: 277-280.

Barr, R.G., Kramer, M.S., Boisjoly, C., McVey-White, L. and Plesset, I.B., 1988. Parental diary of infant cry and fuss behaviour. Archives of Disease in Childhood 63: 380-387. https://doi.org/10.1136/ adc.63.4.380

Biagioli E, Tarasco V, Lingua, C., Moja, L. and Savino, F., 2016. Painrelieving agents for infantile colic. Cochrane Database of Systematic Reviews 9: CD009999. https://doi.org/10.1002/14651858.CD009999

Chau, L., Lau, E., Greenberg, S., Jacobson, S., Yazdani-Brojeni, P., Verma, N. and Koren, G., 2015. Probiotics for infantile colic: a randomized, double-blind, placebo-controlled trial investigating Lactobacillus reuteri DSM 17938. Journal of Pediatrics 166: 74-78. https://doi.org/10.1016/j.jpeds. 2014.09.020

Danielsson, B. and Hwang, C.P., 1985. Treatment of infantile colic with surface active substance (simethicone). Acta Paediatrica Scandinavica 74: 446-450.

De Weerth, C., Fuentes, S. and De Voss, W.M., 2013. Crying in infants. On the possible role of intestinal microbiota in the development of colic. Gut Microbes 4: 416-421. https://doi.org/10.4161/gmic.26041

Dorreh, F., Hashemi, S.M., Salehi, S., Taheri, M. and Almasi-Hashiani, A., 2017. The effect of probiotic in treatment of infantile colic: a randomized clinical trial. International Journal of Pediatrics 5: 5295-5303. https://doi.org/10.22038/ijp.2017.22530.1884

Dryl, R. and Szajewska, H., 2018. Probiotics for management of infantile colic: a systematic review of randomized controlled trials. Archives of Medical Science 14: 1137-1143. https://doi.org/10.5114/ aoms.2017.66055

Fatheree, N.Y., Liu, Y., Ferris, M., Van Arsdall, M., McMurtry, V., Zozaya, M., Cai, C., Rahbar, M.H., Hessabi, M., Vu, T., Wong, C., Min, J., Q Tran, D., Navarro, F., Gleason, W., Gonzalez, S. and Rhoads, J.M., 2016. Hypoallergenic formula with Lactobacillus rhamnosus GG for babies with colic: a pilot study of recruitment, retention, and fecal biomarkers. World Journal of Gastrointestinal Pathophysiology 7: 160-170. https://doi.org/10.4291/wjgp.v7.i1.160 Giglione, E., Prodam, F., Bellone, S., Monticone, S., Beux, S., Marolda, A., Pagani, A., Di Gioia, D., Del Piano, M., Mogna, G. and Bona, G., 2016. The Association of Bifidobacterium breve BR03 and B632 is effective to prevent colics in bottle-fed infants. Journal of Clinical Gastroenterology 50: S164-S167. https://doi.org/10.1097/ MCG.0000000000000693

Guo, Q. and Goldenberg, J.Z., 2019. Probiotics for the prevention of pediatric antibiotic-associated diarrhea. Cochrane Database of Systematic Reviews 4: CD004827. https://doi.org/10.1002/14651858. CD004827.pub4

Helseth, S. and Begnum, S., 2002. A comprehensive definition of infant colic: parents' and nurses' perspectives. Journal of Clinical Nursing 11: 672-680. https://doi.org/10.1046/j.1365-2702.2002.00643.x

Kianifar, H., Ahanchian, H., Grover, Z., Jafari, S., Noorbakhsh, Z., Khakshour, A., Sedaghat, M. and Kianiet, M., 2014. Synbiotic in the management of infantile colic: a randomised controlled trial. Journal of Paediatrics and Child Health 50: 801-805. https://doi. org/10.1111/jpc. 12640 
Lam, J., Barr, R.G., Catherine, N., Tsui, H., Hahnhaussen, C.L., Pauwels, J. and Brant, R., 2010. Electronic and paper diary recording of infant and caregiver behaviors. Journal of Developmental and Behavioral Pediatrics 31: 685-693. https://doi.org/10.1097/ dbp.0b013e3181e416ae

Landgren, K. and Hallström, I., 2011. Parents' experience of living with a baby with infantile colic - a phenomenological hermeneutic study. Scandinavian Journal of Caring Sciences 25: 317-324.

Lucassen, P., 2010. Colic in infants. BMJ Clinical Evidence 11: 0309.

Lucassen, P., Assendelft, W., Van Eijk, J.T.M., Gubbels, J., Douwes, A. and Van Geldrop, W.J., 2001. Systematic review of the occurrence of infantile colic in the community. Archives of Disease in Childhood 84: 398-403. https://doi.org/10.1136/adc.84.5.398

Mai, T., Fatheree, N.Y., Gleeson, W., Liu, Y. and Rhoads, J.M., 2018. Infantile colic: new insights into an old problem. Gastroenterology Clinics of North America 47: 829-844. https://doi.org/10.1016/j. gtc.2018.07.008

Meier, R. and Steuerwald, M., 2007. Review of the therapeutic use of simethicone in gastroenterology. Schweizerische Zeitschrift für Ganzheitsmedizin 19: 380-387.

Metcalf, T.J., Irons, T.G., Sher, L.D. and Young, P.C., 1994. Simethicone in the treatment of infant colic: a randomized multicenter trial. Pediatrics 94: 29-34.

Mi, G.L., Zhao, L., Qiao, D.D., Kang, W.Q., Tang, M.Q. and Xu, J.K., 2015. Effectiveness of Lactobacillus reuteri in infantile colic and colicky induced maternal depression: a prospective single blind randomized trial. Antonie Van Leeuwenhoek 107: 1547-1553. https://doi.org/10.1007/s10482-015-0448-9

Nocerino, R., De Filippis, F., Cecere, G., Marino, A., Micillo, M., Di Scala, C., De Caro, C., Calignano, A., Bruno, C., Paparo, L., Iannicelli, A.M., Cosenza, L., Maddalena, Y., Della Gatta, G., Coppola, S., Carucci, L., Ercolini, D. and Canani R.B., 2020. The therapeutic efficacy of Bifidobacterium animalis subsp. lactis BB-12 in infant colic: a randomised, double blind, placebo-controlled trial. Alimentary Pharmacology and Therapeutics 51: 110-120. https:// doi.org/10.1111/apt.15561

Ong, T., Gordon, M., Banks, S.S.C., Thomas, M.R. and Akobeng, A.K., 2019. Probiotics to prevent infantile colic. Cochrane Database of Systematic Reviews 3: CD012473. https://doi.org/10.1002/14651858. CD012473

Pärtty, A., Lehtonen, L., Kalliomäki, M., Salminen, S. and Isolauri, E., 2015. Probiotic Lactobacillus rhamnosus GG therapy and microbiological programming in infantile colic: a randomized, controlled trial. Journal of Pediatric Research 78: 470-475. https:// doi.org/10.1038/pr.2015.127

Piatek, J., Krauss, H., Ciechelska-Rybarczyk, A., Bernatek, M. WojtylaBuciora, P. and Sommermeyer, H., 2020. In-vitro growth inhibition of bacterial pathogens by probiotics and a synbiotic: product composition matters. International Journal of Environmental Research and Public Health 17: 3332. https://doi.org/10.3390/ ijerph 17093332

Roos, S., Dicksved, J., Tarasco, V., Locatelli, E., Ricceri, F., Grandin, U. and Savino, F., 2013. 454 pyrosequencing analysis on faecal samples from a randomized DBPC trial of colicky infants treated with Lactobacillus reuteri DSM 17938. PLoS ONE 8: e56710. https:// doi.org/10.1371/journal.pone.0056710
Saavedra, J.M., Abi-Hanna, A., Moore, N. and Yolkenet, R.H., 2004. Long-term consumption of infant formulas containing live probiotic bacteria: tolerance and safety. American Journal of Clinical Nutrition 79: 261-267. https://doi.org/10.1093/ajcn/ 79.2.261

Santas, J., Fuentes, M.C., Tormo, R., Escolies, G.R., Lazaro, E. and Cune, J., 2015. Pediococcus pentosaceus CECT 8330 and Bifidobacterium longum CECT 7894 show a trend towards lowering infantile excessive crying syndrome in a pilot clinical trial. International Journal of Pharma and Bio Sciences 6: 458-466.

Sarasu, J.M., Narang, M. and Shah D., 2018. Infantile colic: an update. Indian Pediatrics 55: 979-987.

Savino, F., Bailo, E., Oggero, R., Tullio, V., Roana, J., Carlone, N., Cuffini, A.M. and Silvestro, L., 2005. Bacterial counts of intestinal Lactobacillus species in infants with colic. Pediatric Allergy Immunology 16: 72-75. https://doi.org/10.1111/j.13993038.2005.00207.x

Savino, F., Cordisco, L., Tarasco, V., Calabrese, R., Palumeri, E. and Matteuzziet, D., 2009. Molecular identification of coliform bacteria from colicky breastfed infants. Acta Paediatrica 98: 1582-1588. https://doi.org/10.1111/j.1651-2227.2009.01419.x

Savino, F., Cordisco, L., Tarasco, V., Locatelli, E., Di Gioia, D., Oggero, R. and Matteuzzi, D., 2011. Antagonistic effect of Lactobacillus strains against gas-producing coliforms isolated from colicky infants. BMC Microbiology 11: 157. https://doi.org/10.1186/1471-218011-157

Savino, F., Cordisco, L., Tarasco, V., Palumeri, E., Calabrese, R., Oggero, R., Roos, S. and Matteuzziet, D., 2010. Lactobacillus reuteri DSM 17938 in infantile colic: a randomized, double-blind, placebocontrolled trial. Pediatrics 126: e526-533. https://doi.org/10.1542/ peds.2010-0433

Savino, F., Cresi, F., Pautasso, S., Palumeri, E., Tullio, V., Roana, J., Silvestro, L. and Oggero, R., 2004. Intestinal microflora in breastfed colicky and non-colicky infants. Acta Paediatrica 93: 825-829.

Savino, F., Garro, M., Montanari, P., Galliano, I. and Bergallo, M., 2018. Crying time and ROR $\gamma / F O X P 3$ expression in Lactobacillus reuteri DSM17938-treated infants with colic: a randomized trial. Journal of Pediatrics 192: 171-177. https://doi.org/10.1016/j.jpeds.2017.08.062

Schreck Bird, A., Gregory, P.J., Jalloh, M.A., Risoldi Cochrane, Z. and Hein, D.J., 2017. Probiotics for the treatment of infantile colic: a systematic review. Journal of Pharmacy Practice 30: 366-374. https:// doi.org/10.1177/0897190016634516

Sethi, K.S. and Sethi, J.K., 1988. Simethicone in the management of infant colic. Practitioner 232: 508.

Stone, A.A., Shiffman, S., Schwartz, J.E., Broderick, J.E. and Hufford, M.R., 2003. Patient compliance with paper and electronic diaries. Controlled Clinical Trials 24: 182-199. https://doi.org/10.1016/ s0197-2456(02)00320-3

Sung, V., 2018. Infantile colic. Australian Prescriber 41: 105-110. https://doi.org/10.18773/austprescr.2018.033

Sung, V., D’Amico, F., Cabana, M.D., Chau, K., Koren, G., Savino, F., Szajewska, H., Deshpande, G., Dupont, C., Indrio, F., Mentula, S., Partty, A. and Tancrediet, D., 2018. Lactobacillus reuteri to treat infant colic: a meta-analysis. Pediatrics 141: e20171811. https:// doi.org/10.1542/peds.2017-1811 
Sung, V., Hiscock, H., Tang, M.L.K., Mensah, F.K., Nation, M.L., Satzke, C., Heine, R.G., Stock, A., Barr, R.G. and Wake, M., 2014. Treating infant colic with the probiotic Lactobacillus reuteri: double blind placebo controlled randomized trial. BMJ 348: g2107. https://doi. org/10.1136/bmj.g2107

Szajewska, H., Gyrczuk, E. and Horvath, A., 2013. Lactobacillus reuteri DSM 17938 for the management of infantile colic in breastfed infants: a randomized, double-blind, placebo-controlled trial. Journal of Pediatrics 162: 257-262. https://doi.org/10.1016/j. jpeds.2012.08.004
Tintore, M. and Cune, J., 2017. Probiotic treatment with ab-kolicare causes changes in the microbiota which correlate with a reduction in crying time. International Journal of Pharma and Bio Sciences 8: 281-288. https://doi.org/10.22376/ijpbs.2017.8.1.b281-B288

Tintore, M., Colome, G., Santas, J. and Espadaler, J., 2017. Gut microbiota dysbiosis and role of probiotics in infant colic. Archives of Clinical Microbiology 8: 56. https://doi.org/10.4172/19898436.100056

Wessel, M.A., Jackson, E.B., Harris, G.S. and Detwiler, A.C., 1954. Paroxysmal fussing in infancy, sometimes called colic. Pediatrics 14: 421-435. 
